\title{
Multipotent Polymer Coatings Based on Chemical Vapor Deposition Copolymerization**
}

\author{
By Yaseen Elkasabi, Hsien-Yeh Chen, and Joerg Lahann*
}

The controlled and stable immobilization of one or multiple types of (bio)molecules to a surface has been identified as one of the critical challenges in several emerging research fields, such as the regulation of cell shape,$^{[1]}$ the development of advanced biological assays ${ }^{[2,3]}$ and scaffolds for regenerative medicine ${ }^{[4,5]}$ or the fabrication of increasingly complex micrototal analytical systems ( $\mu$ TAS).$^{[6,7]}$ This is partly motivated by the need for defined surface architectures to simultaneously present multiple biological entities in controlled ratios. While a range of methods have been developed for the immobilization of a single type of biomolecule to artificial substrates, fewer concepts are available for the precise immobilization of multiple biomolecules in a controllable fashion. ${ }^{[8]}$ Recently, we established a suite of surface modification techniques based on chemical vapor deposition (CVD) polymerization of substituted [2.2] paracyclophanes. ${ }^{[9-11]}$ This novel coating technology resulted in a diverse class of functionalized poly $p$-xylylenes containing a wide variety of functional groups such as amines, ${ }^{[12]}$ esters, ${ }^{[13,14]}$ aldehydes, ${ }^{[15]}$ and alcohols. ${ }^{[16]}$ The resulting polymers provide a flexible solution to surface-engineering challenges, as they decouple surface design from bulk properties. ${ }^{[17]}$ In essence, CVD technology is a one-step coating procedure that generates functionalized surfaces without the need for further post-treatments once the films are deposited. $^{[18]}$ In a significant extension of our previous work, we now report on the use of CVD copolymerization to fabricate multipotent and modular coatings. The term "multipotent coating" in this context, refers to a reactive coating that is compatible with the simultaneous presentation of multiple biomolecules in controllable ratios.

Prior to CVD copolymerization, 4-trifluoroacetyl [2.2]paracyclophane (1) and 4-aminomethyl-[2.2]paracyclophane (2) were synthesized from commercially available [2.2]paracyclophane following established synthetic routes. ${ }^{[10,19]}$ CVD copolymerization of $\mathbf{1}$ and $\mathbf{2}$ was then conducted and resulted in a

[*] Prof. J. Lahann, Y. Elkasabi, H. Y. Chen Departments of Chemical Engineering, Materials Science and Engineering, and Macromolecular Science and Engineering University of Michigan 2300 Hayward Street, Ann Arbor, MI 48109 (USA) E-mail: lahann@umich.edu

[*** JL gratefully acknowledges support from the NSF in form of a CAREER grant (DMR-0449462) and a Major Instrumentation Grant (DMR-0420785). The authors thank Dr. Erdogan Gulari, University of Michigan, for use of the fluorescence scanner. Supporting Information is available online from Wiley InterScience or from the author. vacuum-deposited film of copolymer $\mathbf{3}$ on the substrate (Scheme 1).

For CVD copolymerization, mixtures of carefully purified dimers $\mathbf{1}$ and $\mathbf{2}$ were initially sublimated under a reduced pressure of $56 \mathrm{~Pa}$ at $90-100^{\circ} \mathrm{C}$. The sublimation temperatures of $\mathbf{1}$ and $\mathbf{2}$ were sufficiently similar, and so to ensure that the compounds were exposed to comparable sublimation conditions they were placed near each other within the CVD system. Sublimated $\mathbf{1}$ and $\mathbf{2}$ were then transferred to the pyrolysis zone, which was heated to $670{ }^{\circ} \mathrm{C}$ to ensure cleavage of the aliphatic $\mathrm{C}-\mathrm{C}$ bonds, resulting in the corresponding quinodimethanes (monomers). In the last step, monomers were adsorbed on the substrate at approximately $10^{\circ} \mathrm{C}$ and spontaneously polymerized. CVD copolymerization of $\mathbf{1}$ and $\mathbf{2}$ resulted in transparent and topologically uniform polymer films (Figure S2, Supporting Information) with thicknesses between 50 and $200 \mathrm{~nm}$. The film thickness is mainly determined by the total amount of [2.2]paracyclophanes used for polymerization. For instance, the thickness of a film produced by the deposition of $20 \mathrm{mg}$ of equimolar amounts of $\mathbf{1}$ and $\mathbf{2}$ was determined by means of imaging ellipsometry to be $115 \pm 15 \mathrm{~nm}$. Moreover, the multifunctional coatings showed excellent chemical stability in a dry-air environment. No significant change in composition or chemical behavior was found for samples stored in a dry-air atmosphere for several weeks compared to freshly prepared samples. All copolymers as well as the two homopolymers $\mathbf{4}$ and $\mathbf{5}$ remained intact after rinsing with standard solvents such as water, chloroform, acetone, and ethanol.

The elemental composition of the copolymer poly[4-aminomethyl- $p$-xylylene-co-4-trifluoroacetyl- $p$-xylylene-co- $p$-xylylene] (3) was studied by X-ray photoelectron spectroscopy (XPS). XPS is capable of detecting atomic composition within a depth of about $10 \mathrm{~nm} .{ }^{[20]}$ Copolymer 3 was compared to the individual polymers, poly[4-trifluoroacetyl- $p$-xylylene-co- $p$ xylylene] (4) and poly[4-aminomethyl- $p$-xylylene-co- $p$-xylylene] (5, see Table 1).

Characteristic chemical elements detected for the individual polymers 4 and 5, such as oxygen (polymer 4), fluorine (polymer 4), and nitrogen (polymer 5), were simultaneously detected in the copolymer, indicating the presence of both functional groups on the surface. Because nitrogen is only present in the aminomethyl group of polymer $\mathbf{5}$, while fluorine is present in only the ketone function of polymer $\mathbf{4}$, the ratio of the elemental composition of nitrogen and fluorine is a good indicator of the chemical quality of the copolymer films. Using XPS, we found a N/F ratio of 0.332 , which is in good ac- 
(1)

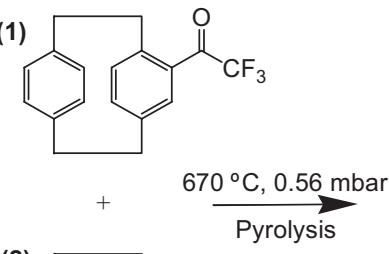

(2)

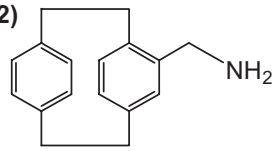<smiles>C=c1ccc(=C)c(C(=O)C(F)(F)F)c1</smiles><smiles>CCOCCOC</smiles><smiles></smiles><smiles>CCc1ccc(C)c(C(=O)C(F)(F)F)c1</smiles><smiles>c1ccccc1</smiles><smiles>Cc1ccc(C)cc1</smiles><smiles>CCc1ccc(C)c(CN)c1</smiles>

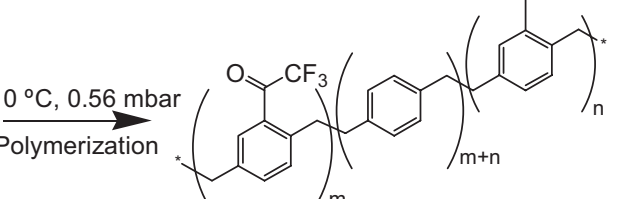

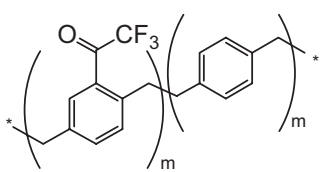

(4)

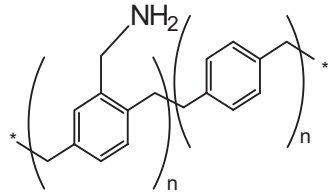

(5)

Scheme 1. The multifunctional polymer (3) accessible by CVD copolymerization of [2.2]paracyclophanes 1 and 2; the structures of the individual polymers 4 and 5 are shown for comparison. (1 mbar $=100 \mathrm{~Pa}$.)

Table 1. High-resolution C1s XPS results for poly[(4-aminomethyl-p-xylylene)-co-(4-trifluoroacetyl-p-xylylene)-co-p-xylylene] (3) prepared with a 1:1 feed ratio, compared to the individual polymers 4 and 5 . BE: binding energy.

\begin{tabular}{lcccccc}
\hline Polymer & & $\mathrm{C}-\mathrm{C}$ & $\mathrm{C}-\mathrm{N}$ & $\mathrm{C}=\mathrm{O}$ & $\pi \rightarrow \pi^{*}$ & $\mathrm{C}-\mathrm{F}$ \\
& $B E[\mathrm{eV}]$ & 285.0 & 286.8 & 288.4 & 291.2 & 292.9 \\
\hline $\mathbf{3}$ & Calculated [\%] & 89.2 & 3.6 & 3.6 & - & 3.6 \\
& Experimental [\%] [a] & 89.5 & 3.1 & 3.3 & $4.3[\mathrm{~b}]$ & 4.1 \\
$\mathbf{4}$ & Calculated [\%] & 81.48 & 0 & 9.26 & - & 9.26 \\
& Experimental [\%] & 80.17 & 0 & 9.29 & 4.2 & 10.54 \\
$\mathbf{5}$ & Calculated [\%] & 94.12 & 5.88 & 0 & - & 0 \\
& Experimental [\%] & 94.27 & 5.73 & 0 & 5.8 & 0 \\
\hline
\end{tabular}

[a] Percentage of total without $\pi \rightleftharpoons \pi *$ applies to all experimental values. [b] Actual value obtained; applies to all $\pi \rightleftharpoons \pi *$ values.

cordance with the theoretically expected ratio of 0.333 . Accordingly, side reactions, such as decomposition of the functional groups, were negligible when pyrolysis temperatures under $670{ }^{\circ} \mathrm{C}$ and working pressures between 40 and $60 \mathrm{~Pa}$ were chosen. Furthermore, XPS revealed no signs of cross-reaction of the aminomethyl and ketone groups under the conditions of CVD polymerization. Table 1 shows the experimental results of the high-resolution C1s XPS for the 1:1 copolymer to be in good agreement with theoretical values. ${ }^{[9,21]}$

To further support the XPS data, we used grazing-angle Fourier-transform IR (FTIR) spectroscopy to assess the structure of copolymers deposited onto a gold substrate. Figure 1 displays IR spectra of polymer films made by copolymerization of $\mathbf{1}$ and $\mathbf{2}$ using varying feed ratios. The spectra range from $100 \%$ of compound $\mathbf{2}$ (trace a) to $100 \%$ of compound $\mathbf{1}$ (trace $\mathrm{g}$ ). All spectra were normalized based on the $\mathrm{C}-\mathrm{H}$ band intensity $\left(2926.1 \mathrm{~cm}^{-1}\right)$, because the $\mathrm{C}-\mathrm{H}$ group is present in each polymer. As the feed ratio changed, the peak intensities of side groups changed accordingly. Due to potential differences in film thickness, we restrained ourselves to qualitative analysis only. The carbonyl stretches at $1716 \mathrm{~cm}^{-1}$, characteristic of the $\mathrm{COCF}_{3}$ groups, were compared to the $\mathrm{C}-\mathrm{H}$ band at $2926.1 \mathrm{~cm}^{-1}$. The carbonyl stretch at $1716 \mathrm{~cm}^{-1}$ is absent in spectrum a. For spectra $b-g$, the carbonyl stretch grows until maximum intensity is attained in spectrum $\mathrm{g}$. The same trend can be observed for the C-F stretches at 1227, 1202, and $1152 \mathrm{~cm}^{-1}$. Similarly, the signal at $977 \mathrm{~cm}^{-1}$ increases as the contribution of the ketone increases, to become a strong signal in the spectrum of pure polymer 4 . This band has been previously reported for similar polymers. ${ }^{[22]}$

Once the fundamental concept of CVD copolymerization of [2.2]paracyclophanes containing different functional groups was established, the structural properties of the resulting copolymers needed to be elucidated in further detail. In principle, CVD copolymerization could result in a true copolymer - with properties distinct from individual polymers 4 and 5 -or in a layered blend of the two polymers.

We used X-ray diffraction (XRD) measurements of thin CVD films before and after annealing to address this question. ${ }^{[23]}$ This approach takes advantage of the fact that poly $(p$ xylylenes) are often semicrystalline polymers with characteristic features in the XRD spectra. ${ }^{[24,25]}$ Since XRD data for polymers $\mathbf{4}$ and $\mathbf{5}$ have not yet been reported, we studied the individual CVD polymers first.

Prior to annealing, no diffraction patterns were obtained for either $\mathbf{4}$ or $\mathbf{5}$, indicating that both films were largely amor- 


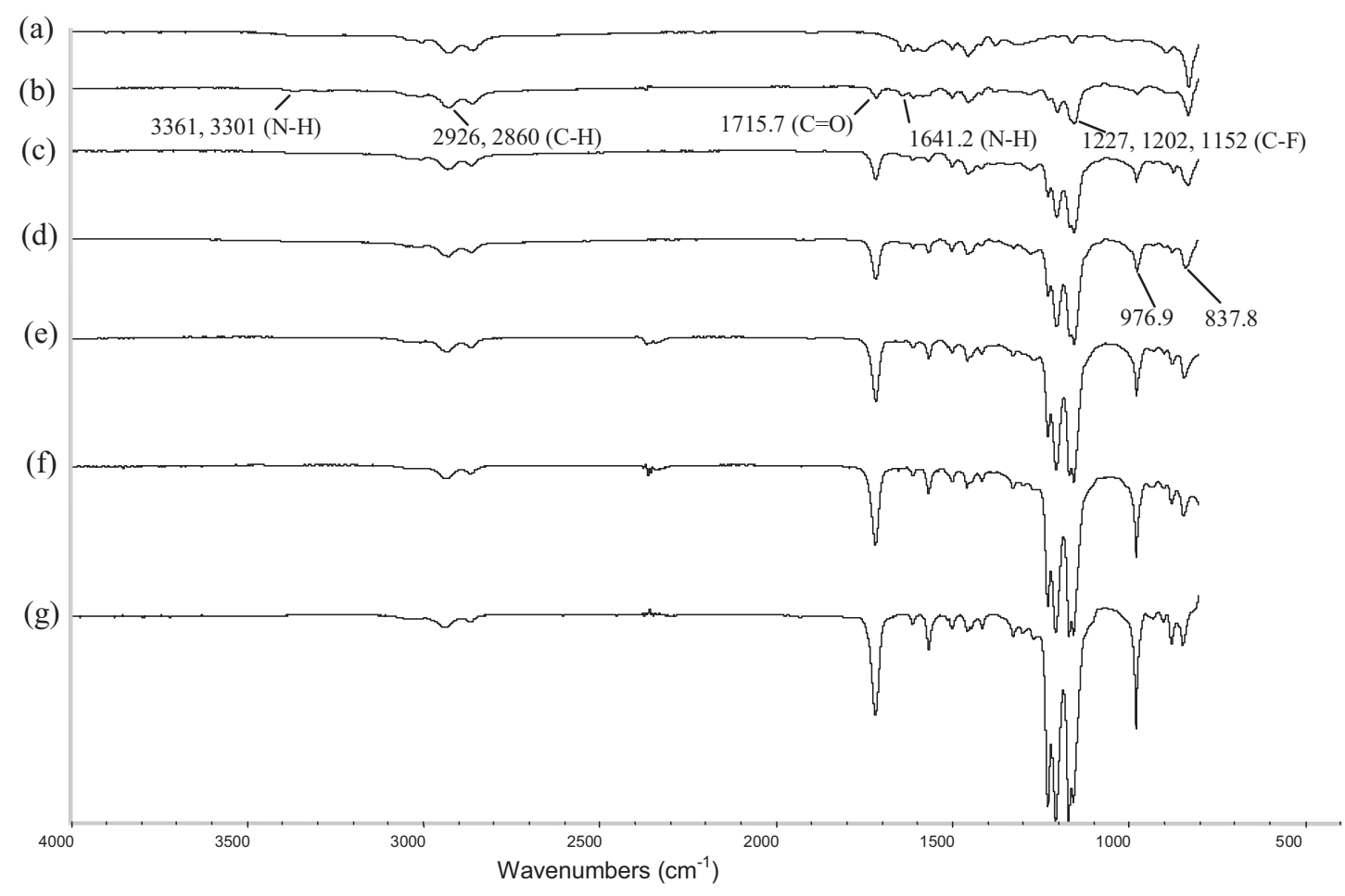

Figure 1. FTIR spectra of (3), obtained from varying molar feed ratios of 1:2. a) $100 \%$ 2, b) 1:5 1:2, c) 1:2, d) 1:1, e) $2: 1$, f) $5: 1$, g) $100 \% 1$.

phous in the as-deposited state. ${ }^{[26]}$ However, after annealing for $14 \mathrm{~h}$ at $120^{\circ} \mathrm{C}$, the trifluoroacetyl-functionalized polymer 4 showed characteristic diffraction patterns, which likely correspond to (020) and (110) planes, and a $d$-spacing greater than that reported for the monoclinic poly( $p$-xylylene) ${ }^{[27]}$ In contrast, the aminomethyl-functionalized polymer 5 remained amorphous. Figure 2 compares diffraction patterns before and after annealing for the individual polymers $\mathbf{4}$ and $\mathbf{5}$ and the copolymers. Exposing the films to a high-temperature environment allows for realignment of functional groups to form crystalline domains. ${ }^{[20]}$ Next, we assessed the structural properties of polymer 3 made by copolymerization of an equimolar feed ratio of $\mathbf{1}$ and $\mathbf{2}$. Based on the FTIR study, we expected this copolymer to contain both components in about the same ratio. If the copolymerization results in a layered blend of individual polymers $\mathbf{4}$ and $\mathbf{5}$, the resulting diffraction pattern should essentially be the superposition of the diffraction patterns of the individual polymers. If, however, the CVD copolymerization results in a true copolymer, a new polymer with a distinct, presumably amorphous structure would be prepared. As shown in Figure 2b, no diffraction patterns were detected for the copolymer, independent of the annealing. This observation also holds for the copolymer made from [2.2]paracyclophanes $\mathbf{1}$ and $\mathbf{2}$ in a feed ratio of 2:1. If a large excess of the ketone is used (feed ratio of 5:1), a small diffraction pattern can be detected after annealing, indicating the onset of a semicrystalline structure. Based on the XRD data, we concluded that the CVD copolymerization results in true copolymers with distinct structural proper- ties, rather than layered blends of the individual polymers 4 and 5.

Finally, we verified that both functional groups contained in copolymer $\mathbf{3}$ are available for further surface modification. The availability of the functional groups for further surface reaction is essential for immobilization of two different types of (bio)molecules. In a proof-of-concept experiment using the approach shown in Scheme 2, the copolymer surface (3) was reacted with two fluorescent ligands that exhibited orthogonal reactivity. The purpose of this experiment was to assess whether or not each functional group remains active and is able to specifically react with the assigned ligands. Fluorescence scanning was used to examine the presence of surfacebound ligands. Figure 3 shows the results of the surface reaction of polymer 3 with Atto 655 and biotin-streptavidin. To avoid reaction between the Atto 655 ligand and the amino groups of streptavidin, a consecutive immobilization scheme was employed: The Atto 655 ligand was immobilized first, followed by the biotin ligand.

We found Atto 655 to bind strongly to the aminomethylcontaining polymer $\mathbf{5}$. In contrast, only negligible amounts of Atto 655 bound to polymer 4 . When reacting the two individual polymers $\mathbf{4}$ and $\mathbf{5}$ with the second ligand, a biotin hydrazide derivative, the biotin ligand was found to bind strongly to polymer 4, but not to $\mathbf{5}$. Fluorescence-labeled streptavidin, a specific binding partner of biotin, was used to visualize the biotin ligands and confirmed selective binding to the biotinmodified coating of polymer 4 . In contrast, streptavidin applied to polymer $\mathbf{5}$, which was not modified with the biotin li- 
(a)

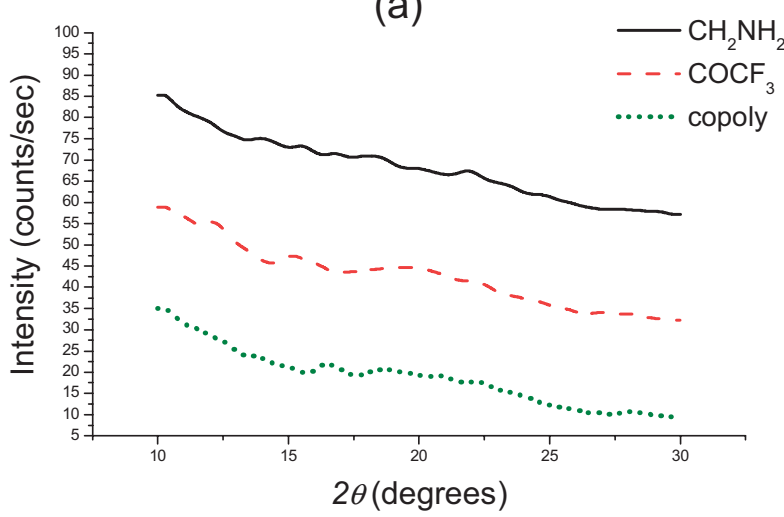

(c)

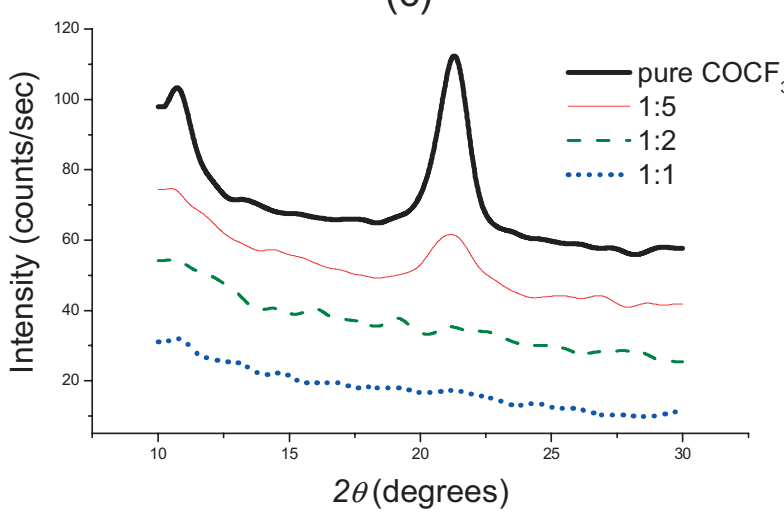

(b)

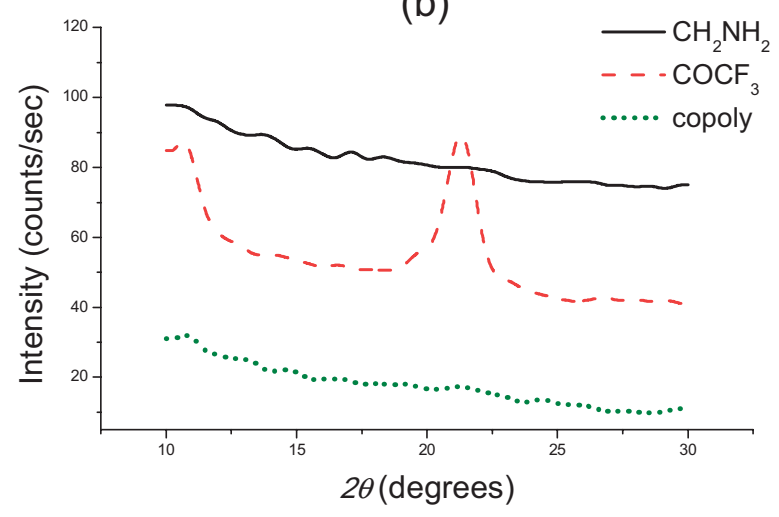

(d)

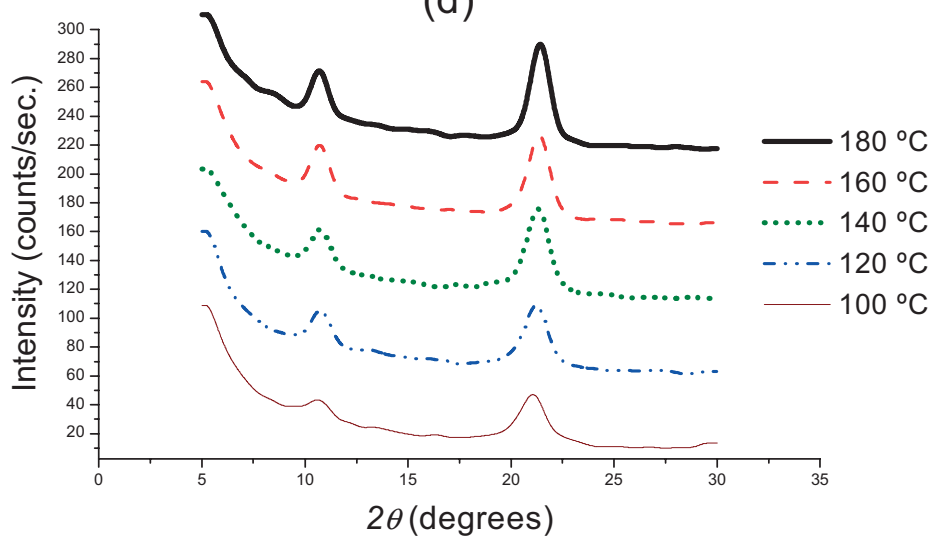

Figure 2. XRD patterns of the individual polymers 4 and $\mathbf{5}$ and the copolymer 3 a) before and b) after annealing at $120^{\circ} \mathrm{C}$. Only polymer 4 exhibits crystallinity after annealing. c) XRD patterns of copolymers with 100\% 1 (black), 1:5 2:1 (red), 1:2 (green), and 1:1 (blue). d) XRD patterns of 4 at various annealing temperatures.

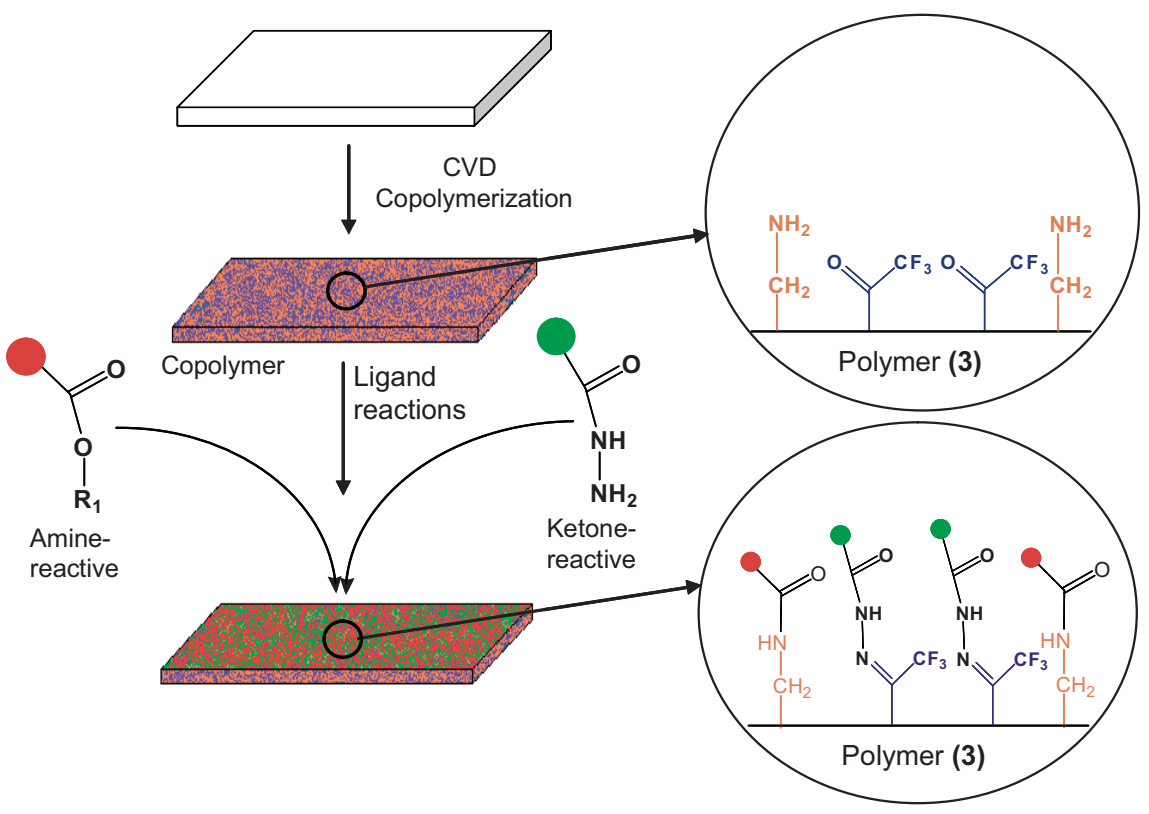

Scheme 2. Schematic outlining the selective reactivity of the multivalent surface. The activated ester only reacts with the aminomethyl group, while the hydrazide group shows selective reactivity towards ketones. gand, did not give any appreciable fluorescence signal. When performing the same procedure on copolymer $\mathbf{3}$, both fluorescence signals were observed simultaneously (Fig. 3). By varying the feed ratios of [2.2]paracyclophanes 1 and $\mathbf{2}$ in the CVD copolymerization, the relative ratio of ligands bound to the surface was varied. The relative increase of starting material 2 resulted in surfaces with increased amounts of Atto-labeled ligands (red color), while a relative increase of compound $\mathbf{1}$ resulted in an increased fluorescence signal indicating more streptavidin-biotin pair bound to the surface. Based on the FTIR spectra (Fig. 1), these trends can be explained by the controlled variation of binding sites available for the two ligands on the surface, which corresponds to the feed ratio of starting materials used during CVD copolymerization. For the 1:1 ratio, the fluorescence mi- 


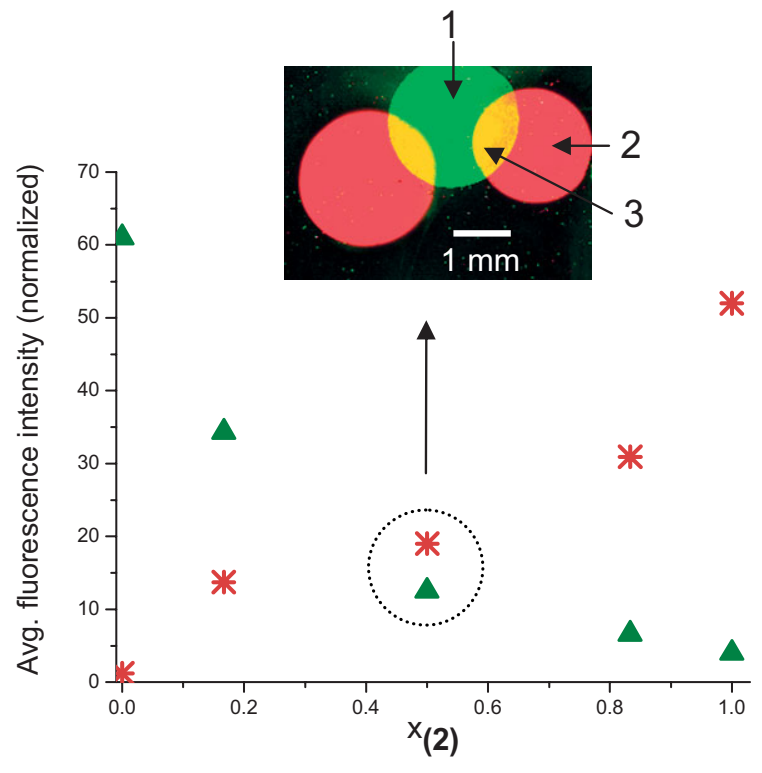

Figure 3. Fluorescence intensities detected on the copolymers versus $x_{(2)}$ (the relative feed concentration of [2.2]paracyclophane $\mathbf{2}$ used for CVD copolymerization). The trends demonstrate ligand immobilization occurs in controlled ratios as a function of increasing relative ratio of the [2.2]paracyclophanes. Inset: Fluorescence micrograph of areas that were reacted with 1) biotin ligand, 2) Atto 655 ligand, or 3) both.

crographs of different reaction areas are shown in the inset of Figure 3 . Area 1 shows copolymer 3 treated only with the biotin ligand (green color), while area 2 is characterized by red fluorescence resulting from surface-immobilization of the Atto-labeled ligand. Where both ligands were allowed to react with the copolymer $\mathbf{3}$ (area 3 ), a yellow color is observed, indicating parallel immobilization of the two ligands.

The herein proposed concept of CVD polymerization of functionalized [2.2]paracyclophanes establishes a general, but simple protocol for preparation of multireactive polymer films. Applicability of the reactive coating to various substrates, such as polymers, metals, or composites, generates a fairly universal platform without relying on broad chemical alteration of the bulk material. ${ }^{[28-30]}$ Although multipotent biointerfaces could in principle be prepared by simultaneously immobilizing mixtures of two different biomolecules through the same functional groups, the exploration of reactive coatings with two orthogonal functional groups bears several potential advantages: 1) surface ratios can be controlled with high precision, because the ligand immobilization reactions can be conducted independently, 2) substantially different biomolecules can be co-immobilized, even if their transport to the surface is substantially different, and 3) the ability to immobilize biomolecules in sequence allows for combinations of biomolecules that would otherwise cross-react with each other. For applications where substantially different biomolecules need to be immobilized in precisely defined ratios, CVD copolymerization may provide a simple access route. Moreover, this study presents a first step towards the establishment of a modular coating design, where the properties of a coating can simply be dialed in by selecting the right combination of building blocks for CVD copolymerization. Such a modular surface design may be of great value for future biomedical devices, high-throughput screening platforms, microfluidic analysis devices, or diagnostic platforms.

\section{Experimental}

CVD Copolymerization: 4-Aminomethyl [2.2]paracyclophane (2) was synthesized from [2.2]paracyclophane (Aldrich) in a two-step synthesis as described elsewhere [19]. 4-Trifluoroacetyl [2.2]paracyclophane (1) was synthesized via Friedel-Crafts acylation of [2.2]paracyclophane [10]. CVD copolymerization was performed using molar mixtures of $\mathbf{1}$ and $\mathbf{2}$ (for the 1:1 feed ratio, we used $38.0 \mu \mathrm{mol}$ each). To control each source independently, the two species were kept in separate feed dishes within the sublimation zone. A pressure of $56 \mathrm{~Pa}$ and temperatures of $90-100^{\circ} \mathrm{C}$ were employed for CVD copolymerization. Under these conditions, the species sublimated and were transferred in a stream of argon carrier gas $(20 \mathrm{sccm})$ to the pyrolysis zone $\left(670^{\circ} \mathrm{C}\right)$. Following pyrolysis, the diradicals were transferred into the deposition chamber, where polymerization occurred. While the wall temperature was adjusted to $120^{\circ} \mathrm{C}$, the silicon, gold, or glass substrates were cooled to $10^{\circ} \mathrm{C}$ to optimize the deposition onto the substrate and to avoid loss of starting material due to wall deposition. Moreover, rotation of the sample holder ensured uniform film deposition. For copolymerization of varying molar feed ratios all reaction conditions were identical, with the exception of the adjusted ratio of the two starting materials. XPS composition of a copolymer with a 1:1 molar feed ratio: C1s: $83.26 \%$ (calc. $84.75 \%$ ), N1s: $2.84 \%$ (calc. $3.05 \%$ ), F1s: $8.54 \%$ (calc. $9.15 \%$ ), O1s: $5.35 \%$ (calc. $3.05 \%$ ); IR (grazing angle $85^{\circ}$ ): 3361.5, 3301.1, 3007.9, 2926.1, 2860.4, 1715.7, 1641.2, 1499.9, 1454.3, 1227.2, 1202.0,1152.4, 976.9, $837.8 \mathrm{~cm}^{-1}$.

$X$-ray Diffraction: To assess the crystallinity of functionalized poly( $p$-xylylene) films, silicon substrates coated with the polymer of interest were examined by wide-angle XRD using a Rigaku $12 \mathrm{~kW}$ high-intensity rotary anode generator. All CVD films were examined both as-deposited and after annealing. The polymer films were annealed for $14 \mathrm{~h}$ in an oven at a temperature of $120^{\circ} \mathrm{C}$, unless specified otherwise.

Surface Characterization: XPS data were recorded on an Axis Ultra X-ray photoelectron spectrometer (Kratos Analyticals, UK) equipped with a monochromatized $\mathrm{Al} \mathrm{K} \alpha \mathrm{X}$-ray source. All spectra were calibrated with respect to the non-functionalized aliphatic carbon with a binding energy of $285.0 \mathrm{eV}$. For XPS imaging, the lens was in hybrid mode, pass energy was set to $160.0 \mathrm{eV}$, and the aperture was $600 \mu \mathrm{m} \times 600 \mu \mathrm{m}$ for all imaging acquisitions. Thicknesses were recorded at a wavelength of $532 \mathrm{~nm}$ using an $\mathrm{EP}^{3}$-SW imaging ellipsometer (Nanofilm Technologie GmbH, Germany). Both nulling (four zones) and mapping experiments were performed at an incident angle of $60^{\circ}$, and an anisotropic Cauchy model was used to model the ellipsometric parameters $\Psi$ and $\Delta$. IR spectroscopy was performed on a Nicolet 6700 spectrometer utilizing the grazing angle accessory (Smart SAGA) at a grazing angle of $85^{\circ}$.

Surface Reaction: Biotin hydrazide- and rhodamine-linked streptavidin were purchased from Pierce Inc., and Atto $655 \mathrm{~N}$-hydroxysuccinimide (NHS) ester was purchased from Sigma Aldrich. All surface reaction experiments were performed on glass substrates. As a control, amine- and ketone-reactive ligands were applied separately to either polymer 4 or 5 . For surface reactions, $1.7 \mu \mathrm{L}$ of reaction buffer was dispensed onto the film, incubated, and rinsed several times. For the copolymers, consecutive surface reactions were conducted. Fluorescence images and intensities were acquired using a GenePix 4000B scanner with $532 \mathrm{~nm}(17 \mathrm{~mW})$ and $635 \mathrm{~nm}(10 \mathrm{~mW})$ lasers. Both excitation wavelengths were scanned simultaneously at $20 \mu \mathrm{m}$ spatial resolution. The colors shown in the scanning images are not true colors, but were chosen for better differentiation. 
Ligand 1: $0.5 \mathrm{~mL}$ of anhydrous dimethylformamide was added to $1 \mathrm{mg}$ Atto $655 \mathrm{NHS}$ ester. From this solution, $3.6 \mu \mathrm{L}$ was diluted with $2 \mathrm{~mL}$ of $0.1 \mathrm{M}$ sodium bicarbonate buffer ( $\mathrm{pH} 8.3$ ) containing $0.02 \%$ $(\mathrm{v} / \mathrm{v})$ Tween 20. NHS esters have limited lifetimes in aqueous solution, so the buffer was applied to the film immediately upon dilution. The solution was incubated for $1 \mathrm{~h}$ at room temperature, after which the surface was rinsed with ethanol for $5 \mathrm{~min}$ and with phosphate-buffered saline (PBS)/Tween solution for $30 \mathrm{~min}$.

Ligand 2: Biotin hydrazide was diluted to $10 \mathrm{~mm}$ in PBS(pH7.4). The solution was gently heated and acid-catalyzed prior to surface application. Incubation times lasted 5-7 $\mathrm{min}$, which was followed by a wash with PBS containing $0.02 \%(\mathrm{v} / \mathrm{v})$ Tween 20 . The area was then treated with rhodamine-linked streptavidin solution $\left(0.075 \mathrm{mg} \mathrm{mL}^{-1}\right.$ in PBS; $0.02 \%(\mathrm{v} / \mathrm{v})$ Tween $20 ; 0.1 \%(\mathrm{w} / \mathrm{v})$ bovine serum albumin) for $10 \mathrm{~min}$. Finally, the entire glass slide was immersed for $1 \mathrm{~h}$ in a PBS/Tween/BSA solution.

Received: November 16, 2005

Final version: March 1, 2006

Published online: May 18, 2006

[1] C. S. Chen, J. L. Alonso, E. Ostuni, G. M. Whitesides, D. E. Ingber, Biochem. Biophys. Res. Commun. 2003, 307, 355.

[2] H. Tani, K. Maehana, T. Kamidate, Anal. Chem. 2004, 76, 6693.

[3] K. Kato, H. Sato, H. Iwata, Langmuir 2005, 21, 7071.

[4] X. Liu, Y. Won, P. X. Ma, J. Biomed. Mater. Res., Part A 2005, 74A, 84.

[5] M. H. Ho, D. M. Wang, H. J. Hsieh, H. C. Liu, T. Y. Hsien, J. Y. Lai, L. T. Hou, Biomaterials 2005, 26, 3197 .

[6] D. R. Reyes, D. Iossifidis, P. A. Auroux, A. Manz, Anal. Chem. 2002, 74, 2623.

[7] P. A. Auroux, D. Iossifidis, D. R. Reyes, A. Manz, Anal. Chem. 2002, 74, 2637.

[8] D. G. Castner, B. D. Ratner, Surf. Sci. 2002, 500, 28.

[9] J. Lahann, R. Langer, Macromolecules 2002, 35, 4380.
[10] J. Lahann, D. Klee, H. Höcker, Macromol. Rapid Commun. 1998, 19, 441.

[11] J. Lahann, R. Langer, Macromol. Rapid Commun. 2001, 22, 968

[12] J. Lahann, H. Höcker, R. Langer, Angew. Chem. Int. Ed. 2001, 40, 726.

[13] J. Lahann, I. S. Choi, J. Lee, K. Jensen, R. Langer, Angew. Chem. Int. Ed. 2001, 40, 3166

[14] J. Lahann, M. Balcells, T. Rodon, J. Lee, I. Choi, K. Jensen, R. Langer, Langmuir 2002, 18, 3632 .

[15] H. Nandivada, H. Y. Chen, J. Lahann, Macromol. Rapid Commun. $\mathbf{2 0 0 5}, 26,1794$.

[16] K. Schürmann, J. Lahann, J. Meyer, H. Klosterhalfen, D. Vorwerk, D. Klee, R. W. Günther, Radiology 2004, 230, 151.

[17] A. Greiner, Trends Polym. Sci. 1997, 5, 12.

[18] D. Klee, N. Weiss, J. Lahann, in Paracyclophanes (Ed: H. Hopf), Wiley-VCH, Weinheim, Germany 2004

[19] V. I. Rozenberg, T. I. Danilova, E. V. Sergeeva, I. A. Shouklov, Z. A. Starikova, H. Hopf, K. Kuhlein, Eur. J. Org. Chem. 2003, 432.

[20] Biomaterials Science: An Introduction to Materials in Medicine (Eds: B. D. Ratner, A. S. Hoffman, F. J. Schoen, J. E. Lemons), Academic, San Diego, CA 1996.

[21] D. Briggs, G. Beamson, Anal. Chem. 1992, 64, 1729.

[22] C. H. Cheng, E. M. Pearch, J. Polym. Sci., Polym. Chem. Ed. 1980, $18,1883$.

[23] B. D. Cullity, S. R. Stock, Elements of X-ray Diffraction, 3rd Ed., Prentice-Hall, Englewood Cliffs, NJ 2001.

[24] S. Isoda, M. Tsuji, M. Ohara, A. Kawaguchi, Polymer 1983, 24, 1155.

[25] W. D. Niegisch, J. Appl. Phys. 1966, 37, 4001.

[26] All films were deposited at $10^{\circ} \mathrm{C}$.

[27] J. J. Senkevich, S. B. Desu, V. Simkovic, Polymer 1999, 41, 2379.

[28] K. Y. Suh, R. Langer, J. Lahann, Adv. Mater. 2004, 16, 1401.

[29] H. Y. Chen, J. Lahann, Anal. Chem. 2005, 77, 6909.

[30] J. Lahann, M. Balcells, H. Lu, T. Rodon, K. F. Jensen, R. Langer, Anal. Chem. 2003, 75, 2117. 